\title{
DIAGNÓSTICO PELA DERMATOSCOPIA DE DOENÇA DE BOWEN EM PACIENTE HIV POSITIVO
}

\author{
Bárbara Agonio', Alex Panizza Jálkh', Maiko Ramos Maia², Laryssa Madeira de Araújo' \\ 'Fundação de Medicina Tropical do Amazonas/Tropical Medicine Foundation, Dr. Heitor Vieira Dourado, Brasil \\ ${ }^{2}$ Hospital Universitário Getúlio Vargas/Universitary Hospital, Amazonas, Brasil
}

\begin{abstract}
RESUMO - A doença de Bowen é uma forma de carcinoma espinocelular intraepidérmico. Acontece em qualquer parte da pele, porém as áreas fotoexpostas são as mais prevalentes. Pode estar associado a imunossupressão.Paciente masculino, 46 anos, com diagnóstico de SIDA, último CD4 de 55 células. Relata aparecimento de lesão em abdome, de evolução de seis meses. Ao exame, placa numular, eritematosa, não descamativa em região de flanco direito. As hipóteses diagnósticas foram de ceratose seborréica irritada, eczema numular ou melanoma amelanótico. À dermatoscopia, observou-se agrupamentos de vasos glomerulares e escamas brancas. Deste modo, levantou-se a hipótese de se tratar de doença de Bowen. A histopatologia foi compatível com doença de Bowen. A dermatoscopia é uma técnica não invasiva, que permite a visualização morfológica de estruturas não observadas a olho nu. Na DB, caracteriza-se por presença de estruturas vasculares (vasos glomerulares ou pontilhados) e escamas na superfície. Neste caso, a dermatoscopia foi essencial para repensar a hipótese diagnóstica inicial da lesão. Mesmo na lesão não-pigmentada, a dermatoscopia vêm se mostrando uma arma importante no exame clínico do paciente.
\end{abstract}

PALAVRAS-CHAVE - Dermatoscopia; Doença de Bowen; Infecção por HIV.

\section{DERMOSCOPY FOR DIAGNOSIS OF BOWEN'S DISEASE IN HIV POSITIVE PATIENTS}

\begin{abstract}
Bowen's disease (BD) is a form of intraepidermal squamous carcinoma. It occurs in any part of the skin, however the sun-exposed areas are the most prevalent. It might be associated with immunosuppression. A male patient, 46 years-old, diagnosed with AIDS, 55 CD4 cells $/ \mathrm{mm} 3$. He has reported onset of injury in abdomen, with evolution of six months. On examination, it was nummular plate, erythematous, scaly, in the right flank. The possible diagnoses were seborrheic keratosis irritation, nummular eczema or amelanotic melanoma. From the dermoscopy, it was observed clusters of glomerular blood vessels and white scales. Thus arose the possibility of Bowen's disease. Histopathology was compatible with BD. Dermatoscopy is a noninvasive technique that allows the visualization of morphological structures not visible to the naked eye. In the BD, it is characterized by the presence of vascular structures (glomerular or dotted vessels) and scales on the surface. In this case, dermoscopy was essential to rethink the initial diagnosis of the lesion. Even in non-pigmented lesions, dermoscopy have shown to be an important weapon in the clinical examination of the patient.
\end{abstract}

KEY WORDS - Bowen's Disease; Dermoscopy; HIV infections.

Conflitos de interesse: Os autores declaram não possuir conflitos de interesse.

No conflicts of interest.

Suporte financeiro: $O$ presente trabalho não foi suportado por nenhum subsídio ou bolsa.

No sponsorship or scholarship granted.

Recebido/Received - Novembro/November 2014; Aceite/Accepted - Dezembro/December 2014 


\section{Dermatoscopia}

Dr. ․ Bárbara Agonio

Fundação de Medicina Tropical do Amazonas Dr. Heitor Vieira Dourado

Brasil

E-mail: babiagonio@hotmail.com

\section{INTRODUÇÃO}

A doença de Bowen (DB) é uma forma de carcinoma espinocelular intraepidérmico. Acontece em qualquer parte da pele, porém as áreas fotoexpostas são as mais prevalentes'. Além da exposição à radiação ultravioleta, a exposição a arsênio também está associada $^{2}$, imunossupressão, radiação ionizante ${ }^{3}$ e pode estar associado com HPV tipo 16 e 33', A DB não costuma apresentar regressão espontânea. Deve ser diferenciada do líquen simples crônico, psoríase, e outras dermatoses papuloescamosas ${ }^{2}$, ceratoses actínicas, ceratose seborréica irritada, eczema numular ou melanoma amelanótico ${ }^{3}$. A dermatoscopia pode ser usada para ajudar no diagnóstico da DB pigmentada ou não ${ }^{4}$. Mostraremos um caso de DB cujo diagnóstico só foi aventado após a dermatoscopia.

\section{CASO CLÍNICO}

Paciente masculino, 46 anos, com diagnóstico de SIDA, realizando tratamento com terapia antirretroviral, sendo o último CD4 de 55 células $/ \mathrm{mm}^{3}$. Vem à consulta no ambulatório de dermatologia por aparecimento

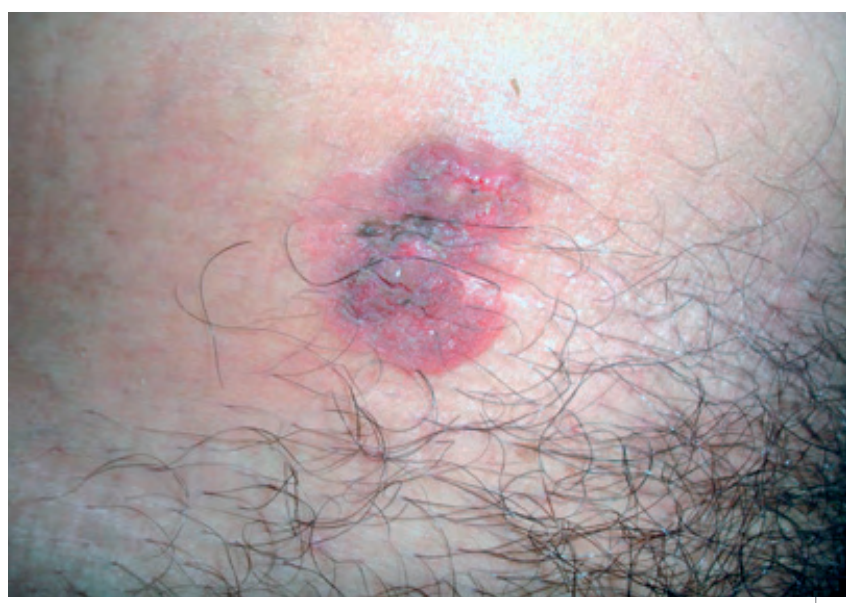

Fig 1 - Placa numular, eritematosa, não descamativa no flanco direito.

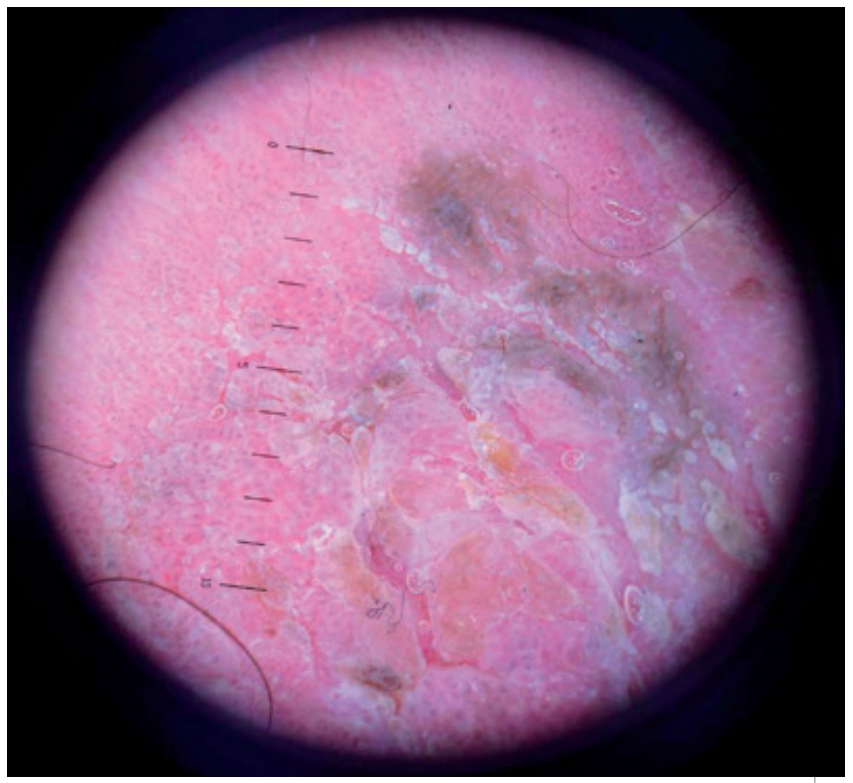

Fig 2 - Aspecto à dermatoscopia: agrupamentos de vasos glomerulares e escamas brancas.

de lesão em abdome, de evolução de seis meses. Relatava leve prurido local. Ao exame, tratava-se de uma placa numular, eritematosa, não descamativa em região de flanco direito (Fig. 1). As hipóteses diagnósticas pensadas foram de ceratose seborréica irritada, eczema numular ou melanoma amelanótico. Realizada a dermatoscopia, observou-se agrupamentos de vasos glomerulares e escamas brancas (Figs. 2 e 3). Deste modo, levantou-se a hipótese de doença de Bowen. Foi realizada biópsia incisional da lesão, e a histopatologia mostrou epiderme com células com núcleos pleomórficos, perda da polaridade e frequentes figuras de mitose, sem alteração da junção dermoepidérmica. Fechando o diagnóstico para doença de Bowen. Posteriormente, realizou-se a exérese completa da lesão.

\section{DISCUSSÃO}

A dermatoscopia é uma técnica não-invasiva, que permite a visualização morfológica de estruturas não observadas a olho $\mathrm{nu}^{5}$. Pode ser usada para auxiliar no 


\section{Dermatoscopia}

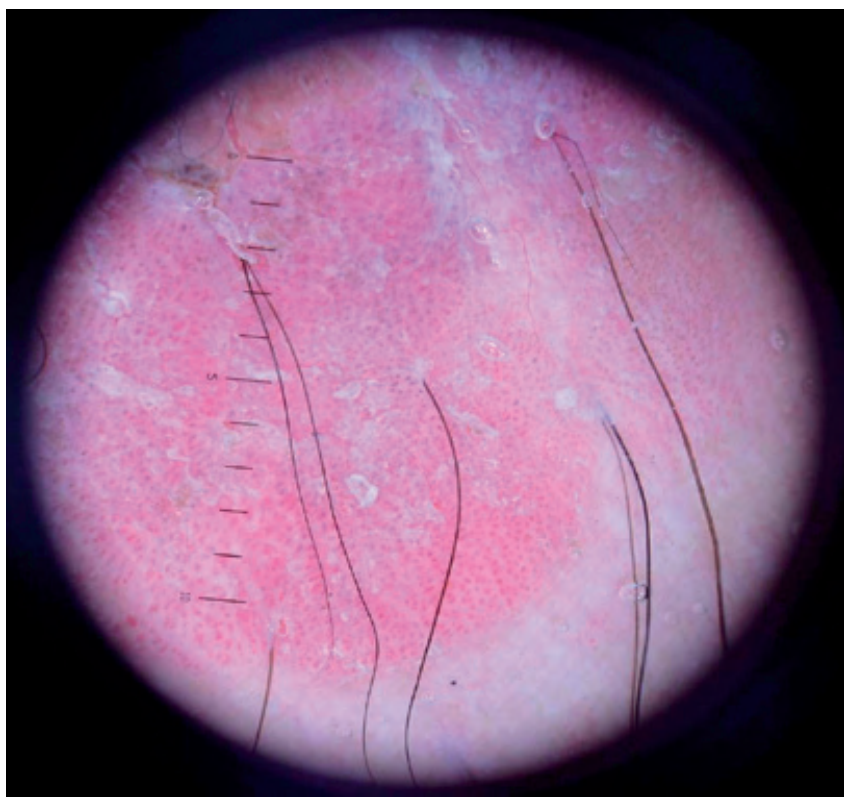

Fig 3 - Outro aspecto da lesão à dermatoscopia.

diagnóstico da DB pigmentada ou não ${ }^{4}$. Caracteriza-se por presença de estruturas vasculares (vasos glomerulares ou pontilhados) em $96 \%$ dos $\operatorname{casos}^{5}$ e escamas na superfície em $90 \%$ dos casos $^{4}$. Essas alterações são características específicas da $\mathrm{DB}^{6}$ e foram observadas na lesão deste paciente.

Os vasos glomerulares representam uma variação dos vasos pontilhados, distribuídos em grupos, mimetizando o aparelho glomerular do rim $^{7}$. Eles correspondem a capilares tortuosos e dilatados da papila dérmica, quando vistos na histopatologia ${ }^{7}$. $O$ arranjo linear ou agrupado dos vasos glomerulares é uma pista específica da doença de Bowen². Os vasos pontilhados podem ser encontrados na psoríase, verrugas virais, acantoma de células claras. Esses vasos também são notados no melanoma amelanótico, porém o arranjo linear e a presença de queratina, pode ajudar a distinção entre eles².

As escamas são brancas ou amareladas e opacas, pode ter aparência queratósica, e cobre algumas áreas do tumor ${ }^{8}$. Pode existir também um halo brancacento em torno dos vasos: um sinal de queratinização?.

Neste caso, a dermatoscopia foi essencial para repensar a hipótese diagnóstica inicial da lesão. Mesmo na lesão não-pigmentada, a dermatoscopia vêm se mostrando uma arma importante no exame clínico do paciente.

\section{REFERÊNCIAS}

1. Kutlubay Z, Engin B, Zara T, Tüzün Y. Anogenital malignancies and premalignancies: Facts and controversies. Clin Dermatol. 2013; 31:362-73.

2. Burns T, Breathnach S, Cox N, Griffi C. Rook's. Textbook of Dermatology. 8th ed. Oxford:Wiley-Blackwell;2010.

3. Wolff K, Goldsmith L, Katz S, Gilchrest B, Paller A, Leffell D. Fitzpatrick's. Dermatology in General Medicine. $7^{\text {th }}$ ed. Philadelphia: McGraw-Hill Medical; 2008.

4. Gencoglan G, Ozdemir F. Nonmelanoma Skin Cancer of the Head and Neck. Clinical Evaluation and Histopathology. Facial Plast Surg Clin N Am. 2012; 20: 423-35.

5. Mun JH, Kim SH, Jung DS, Ko HC, Kwon KS, Kim MB. Dermoscopic features of Bowen's disease in Asians. J Eur Acad Dermatol Venereol. 2010; 24: 805-10.

6. Zalaudek I, Kreusch J, Giacomel J, Ferrar G, Catricalà $C$, Argenziano $G$. How to diagnose nonpigmented skin tumors: A review of vascular structures seen with dermoscopy. Part II. Nonmelanocytic skin tumors. J Am Acad Dermatol. 2010; 63: 377-86.

7. Zalaudek I, Argenziano G, Leinweber B, Citarella L, Hofmann-Wellenhof R, Malvehy J, et al. Dermoscopy of Bowen's disease. Br J Dermat. 2004; 150: 1112-6.

8. Fargnoli MC, Kostaki D, Piccioni A, Micantonio T, Peris K. Dermoscopy in the diagnosis and management of non-melanoma skin cancers. Eur J Dermatol. 2012; 22(4): 456-63.

9. Cameron A, Rosendahl C, Tschandl P, Riedl E, Kittler H. Dermatoscopy of pigmented Bowen's disease. J Am Acad Dermatol. 2010; 62: 597-604.

10. Henquet, CJ. Anogenital malignancies and pre-malignancies. J Eur Acad Dermatol Venereol. 2011; 25: 885-95. 